\title{
GMOS IFU Observations of the Gas Kinematics in the Radio Galaxy Arp 102B
}

\author{
Guilherme d. S. Couto ${ }^{1}$, Thaisa Storchi-Bergmann ${ }^{1}$, \\ Rogemar A. Riffel ${ }^{1}$, D. J. Axon ${ }^{2}$, and A. Robinson ${ }^{2}$ \\ ${ }^{1}$ Universidade Federal do Rio Grande do Sul, IF, CP 15051, Porto Alegre 91501-970, RS, Brazil \\ Email: guilherme.couto@ufrgs.br \\ ${ }^{2}$ Physics Department, Rochester Institute of Technology, NY 14623, USA
}

Keywords. galaxies: nuclei, galaxies: active, galaxies: individual (Arp 102B), radio continuum: galaxies, galaxies: kinematics and dynamics

The goal of this work is to map the gas excitation and kinematics in the inner $\sim 2$ kiloparsecs of the radio-galaxy Arp 102B. Though being classified as an E0 galaxy, Arp 102B shows a nuclear gas spiral (Fathi et al., in preparation). Previous studies of the gas kinematics in nuclear spirals have led to the conclusion that these structures usually trace gas inflows (Fathi et al. 2006; Storchi-Bergmann et al. 2007; Riffel et al. 2008). We have used integral field spectroscopy obtained with GMOS instrument of the Gemini North telescope to investigate the nature of the nuclear spiral arms. The spectra cover the wavelength range $4400-7300 \AA$ over a field of view of $5 .{ }^{\prime \prime} 5 \times 3 . " 9(2.7 \mathrm{kpc} \times 1.9 \mathrm{kpc})$. The $\mathrm{H} \alpha$ flux map from our IFU spectroscopy shows that the spiral arms extend up to at least $1 \mathrm{kpc}$ from the nucleus. We note the presence of the spiral arms in emission-line maps of $\mathrm{H} \alpha, \mathrm{H} \beta,[\mathrm{O}$ III] $\lambda 5007,[\mathrm{O} \mathrm{I}] \lambda 6300,[\mathrm{~N} \mathrm{II}] \lambda 6585$, and [S II] $\lambda 6717$, with the eastern arm better defined and extending farther from the nucleus than the one to the west. Contours from a $8.4 \mathrm{GHz}$ radio image (Caccianiga et al. 2001) show that the radio structure correlates with the flux distributions of the eastern spiral arm. The highest $\mathrm{H} \alpha / \mathrm{H} \beta$ flux ratios (i.e., the highest reddening) are observed $\sim 0 .^{\prime \prime} 3$ southwest of the nucleus and to the west (at the location of the less-extended spiral arm), while no reddening is observed along the eastern spiral arm. The $[\mathrm{N} \mathrm{II}] \lambda 6584 / \mathrm{H} \alpha$ flux ratio is almost constant at $\sim 1$ along the spiral arms. The ratio of the [S II] emission lines give peak density values of $\sim 900 \mathrm{~cm}^{-3}$ around $0 .{ }^{\prime \prime} 3$ southwest of the nucleus (at the location of the highest reddening). The [O III $] \lambda 5007 / \mathrm{H} \beta$ flux ratio shows values typical of AGN (LINERs) with peak values of $\sim 4$ at the nucleus and towards the southwest and west, and lowest values of $\sim 1.3$ to the northeast and east, suggesting some contribution from star-formation at the latter location. Velocity dispersion maps show high dispersions $\left(\sim 270 \mathrm{~km} \mathrm{~s}^{-1}\right) \sim 0 .{ }^{\prime \prime} 3$ southwest of the nucleus at the location of highest density and reddening, decreasing outwards to $\sim 150 \mathrm{~km} \mathrm{~s}^{-1}$. Velocity centroid maps show redshifts approximately to the west and blueshifts approximately to the east. The radio structure seems to correlate with the blueshifted [O III] emission, suggesting that the blueshifts are due to outflow along the eastern spiral arm. Further details of the present study will be published in a forthcoming paper (Couto et al. 2010, in preparation).

\section{References}

Caccianiga, A., March, M. J. M., Thean, A., \& Dennett-Thorpe, J. 2001, MNRAS, 328, 867

Fathi, K., et al. 2006, ApJ, 641, L25

Storchi-Bergmann, T., et al. 2007, ApJ, 670, 959

Riffel, R. A., et al. 2008, MNRAS, 385, 119 